\title{
Comparison of Measured and Simulated Acoustic Signatures for a Full-Scale Aircraft with and without Airframe Noise Abatement
}

\author{
Mehdi R. Khorrami \\ NASA Langley Research Center \\ Patricio A. Ravetta \\ AVEC Inc. \\ David P. Lockard \\ NASA Langley Research Center \\ Benjamin Duda, Ryan Ferris \\ Exa Corp.
}

$24^{\text {th }}$ AIAA/CEAS Aeroacoustics Conference

Atlanta, Georgia

June 25-29, 2018 


\section{Validation of Full-Scale Airframe Noise Simulations}

\section{$\square$ Goals}

- Extend application of simulation-based airframe noise prediction to full-scale, complete aircraft with extreme geometric detail

- Evaluate aeroacoustic performance of main landing gear (MLG) noise reduction (NR) and Adaptive Compliant Trailing Edge (ACTE) technologies on a G-III aircraft

- Use extensive airframe noise flight test data to benchmark/validate simulation results

- Assess capabilities and shortcomings of selected computational methodology

$\square$ Test Aircraft

- SubsoniC Research Aircraft Testbed (SCRAT/804)

$>$ ACTE flaps without and with MLG fairings

- Baseline G-III aircraft (808)

$>$ Flown in baseline configuration (Fowler flaps, no gear treatments)
NASA 804 (SCRAT)
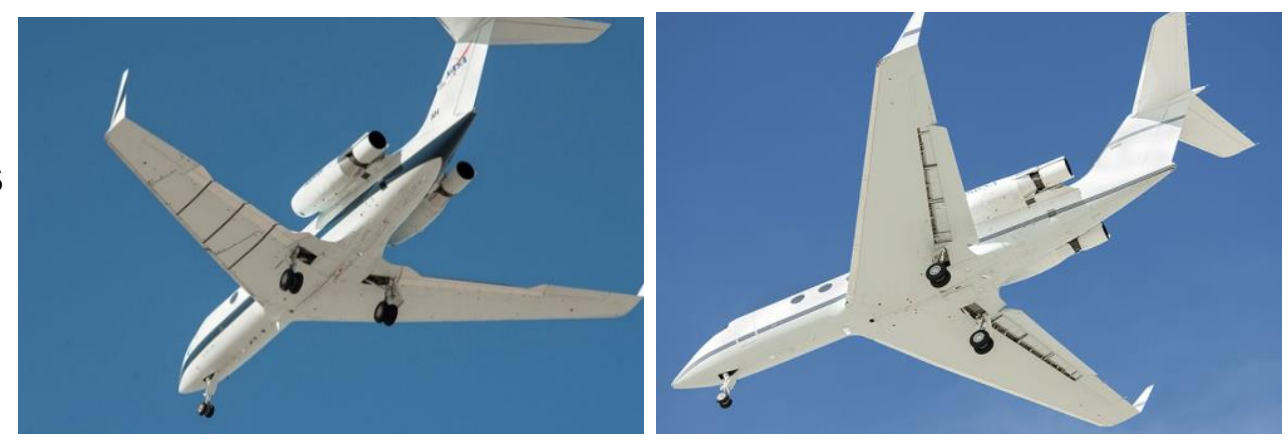
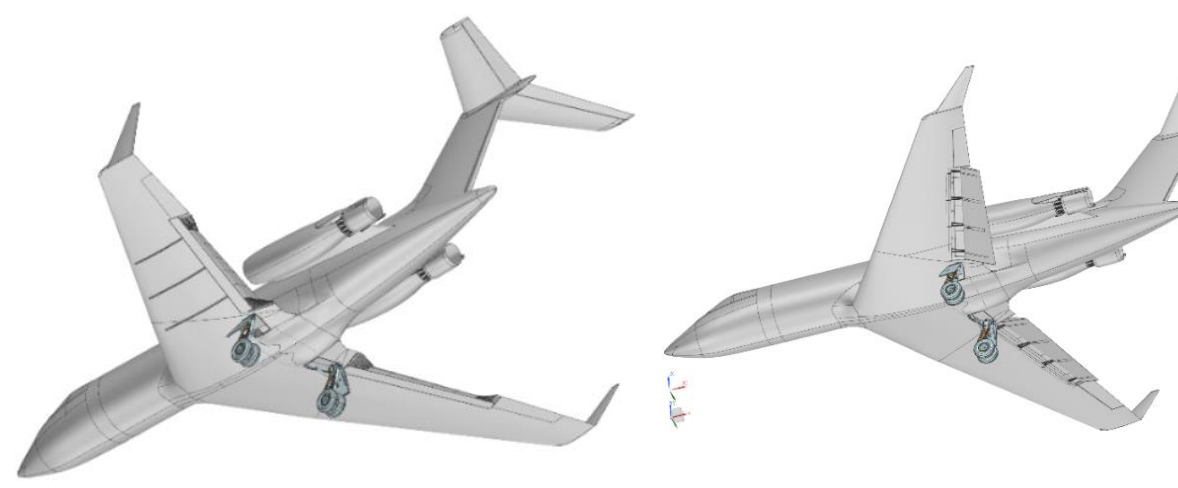


\section{Simulated NR Technologies}

\section{Adaptive Compliant Trailing Edge (ACTE)}

- Technology developed jointly by the U. S. Air Force Research Laboratory (AFRL), FlexSys, Inc., and the NASA ERA project

$>$ Eliminates flap side edges and bracket assemblies

\section{MLG NR Technologies}

- MLG fairings

$>$ Total of 11,332 drilled holes of $D=0.080 "(2 \mathrm{~mm})$

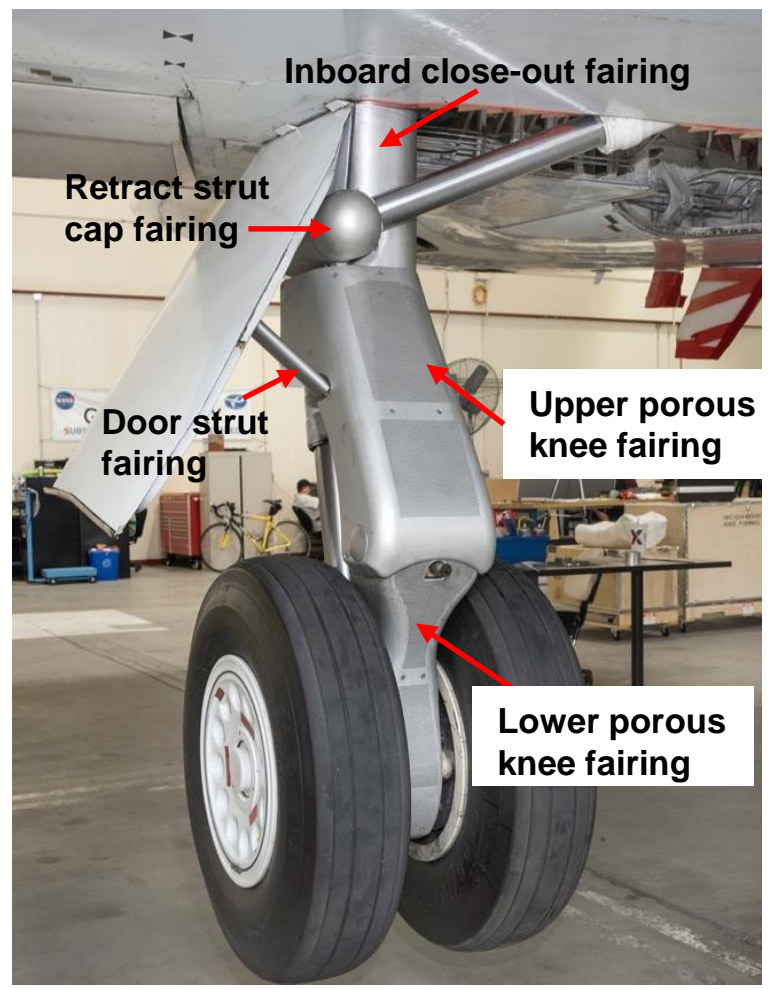

MLG fairings

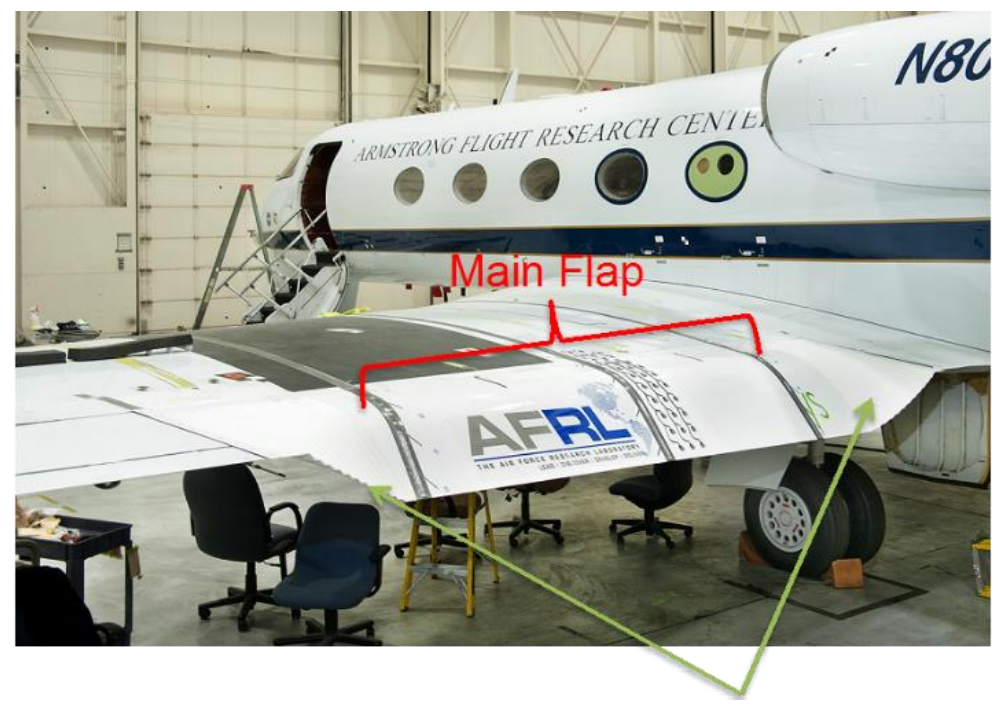

Transition surfaces

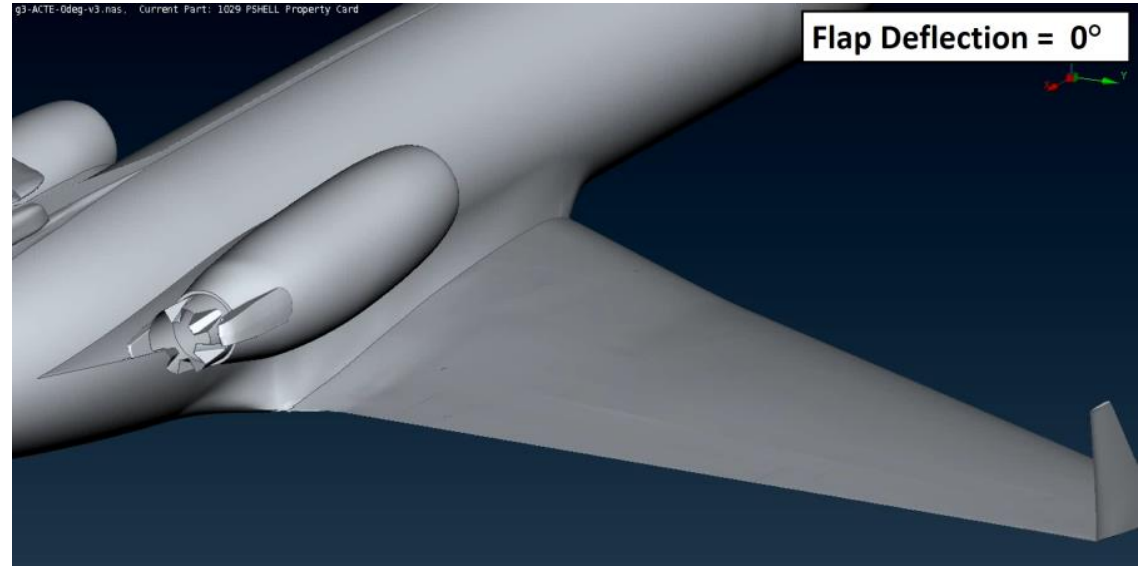

ACTE flap 


\section{Data Sets Used for Simulation Benchmarking}

$\square$ First flight test (Aug. - Oct. 2016)

- Evaluated aeroacoustic performance of ACTE technology

$>$ Microphone array and in-flight steady surface pressure measurements

$>$ Preliminary acoustic measurements for baseline configurations

$\square$ Second flight test (Aug. - Oct. 2017)

- Evaluated acoustic performance of MLG and cavity NR concepts with ACTE flaps

$>$ Microphone array and in-flight steady surface pressure measurements

$>$ Additional acoustic measurements of the baseline configurations

$\square$ Nominal speed of $150 \mathrm{kts}$

$\square$ Engines set at "ground idle"

All simulations performed with Exa's PowerFLOW ${ }^{\circledR}$

\section{Initial Simulations}

- Mostly performed prior to first flight test

- Conducted at medium spatial resolution

$>$ Grid sizes $3 \times 10^{9}$ to $4 \times 10^{9}$ voxels

$>\mathrm{M}=0.228, \mathrm{AOA}=6^{\circ}, \mathrm{Re}=10.5 \times 10^{6}(\mathrm{MAC})$

- Used to optimize design of MLG fairings prior to PDR and CDR

- Used as "blind test" to assess predictive capability of computational approach

$>$ Pressures on aircraft solid surface used in FWH propagation

$>$ Farfield noise spectra computed for single microphone at array center

\section{$\square$ Post $1^{\text {st }}$ Flight Simulations}

- Conducted at fine spatial resolution

$>\mathrm{M}=0.228, \mathrm{Re}=10.5 \times 10^{6}(\mathrm{MAC})$

$>$ Aircraft AOAs matched flight test data

$>$ Pressures on aircraft solid and permeable surfaces used in FWH propagation

$>$ Integrated farfield noise spectra computed from synthetic array data

- Simulations ongoing for various configurations 


\section{Array Data Processing}

\section{a Flight Test}

- Based on time-domain CLEAN technique in AVEC's phased array software suite

- $0.5 \mathrm{~s}$ record corresponding to $\pm 50 \mathrm{ft}$ from array center (90으. overhead)

- Data corrected for temperature and relative humidity (lossless state)

- Scaled to an altitude of $394 \mathrm{ft}(120 \mathrm{~m})$ based on spherical spreading for pressure $\left(\mathrm{p}^{\prime 2} \sim 1 / \mathrm{r}^{2}\right)$
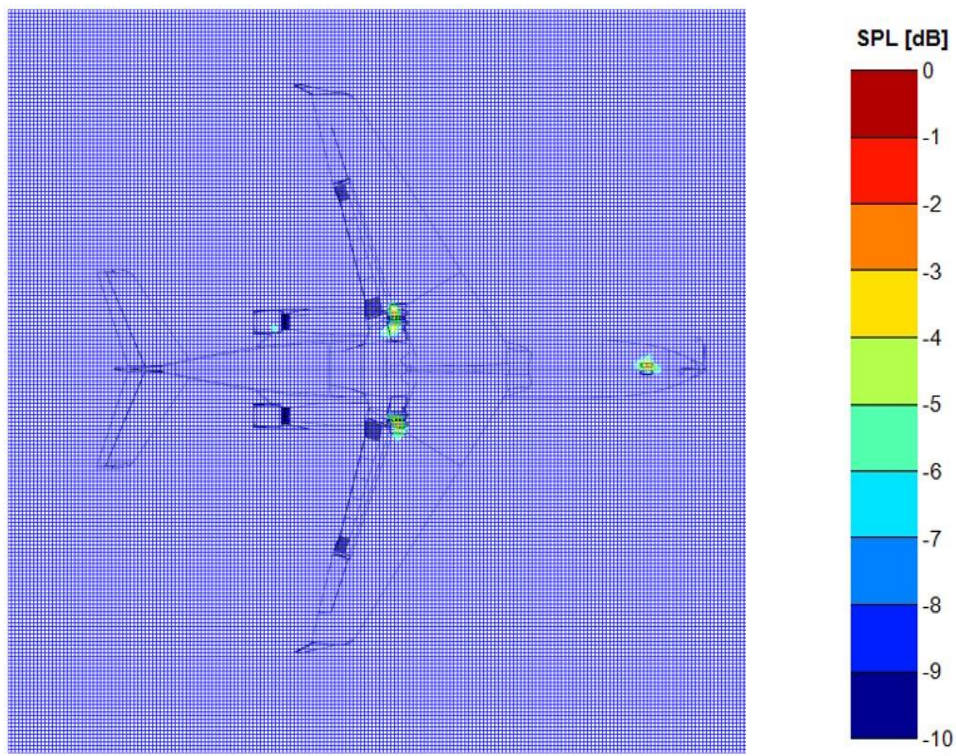

Fine Grid, 201x201, 6 in. resolution

\section{口 Simulations}

- Based on frequency-domain CLEAN technique in AVEC's phased array software suite

- Approx. $1.5 \mathrm{~s}$ record for 90ำ (overhead)

- No atmospheric attenuation needed

- Scaled to an altitude of $394 \mathrm{ft}(120 \mathrm{~m})$ based on spherical spreading for pressure $\left(\mathrm{p}^{\prime 2} \sim 1 / \mathrm{r}^{2}\right)$

Beamform map for 808 aircraft: Fowler flap 39일 landing gear deployed 


\section{Blind Test Comparison}

ACTE flap, gear effects

\section{Fowler flap} deflection effects

\section{Flight test (integrated)}
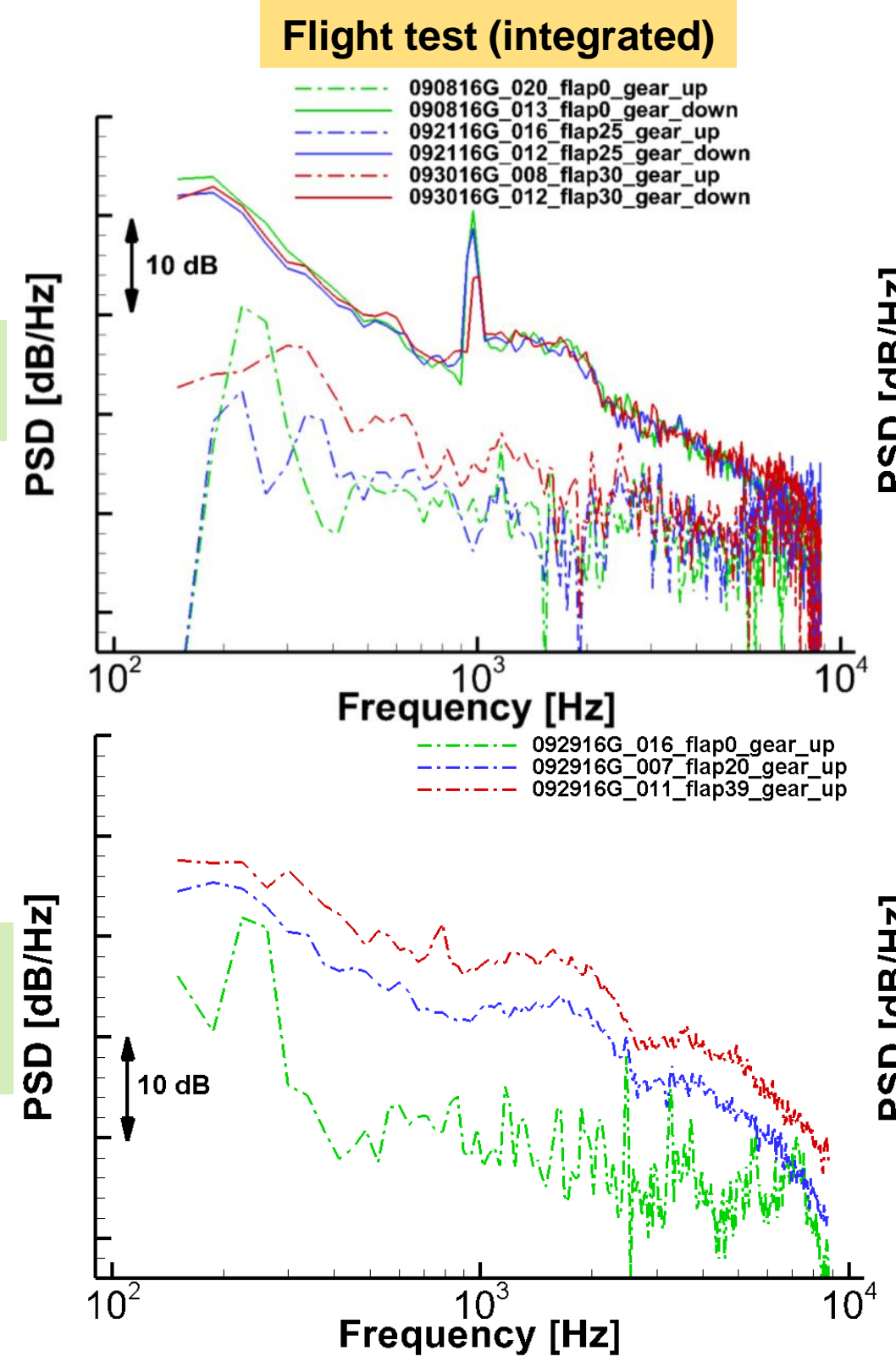

Simulations (array center position)
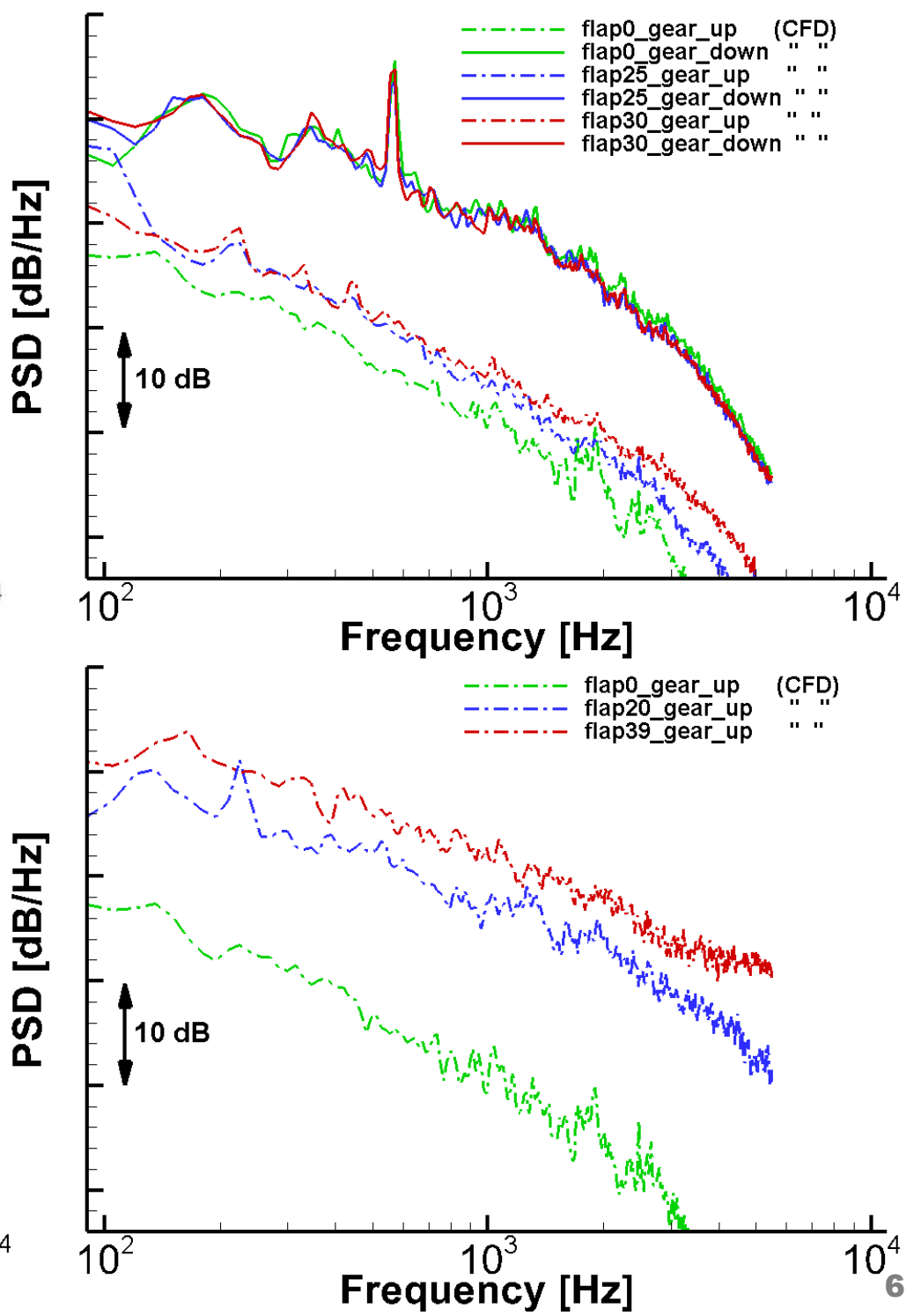


\section{Blind Test Comparison}

\section{Flight test (integrated)}

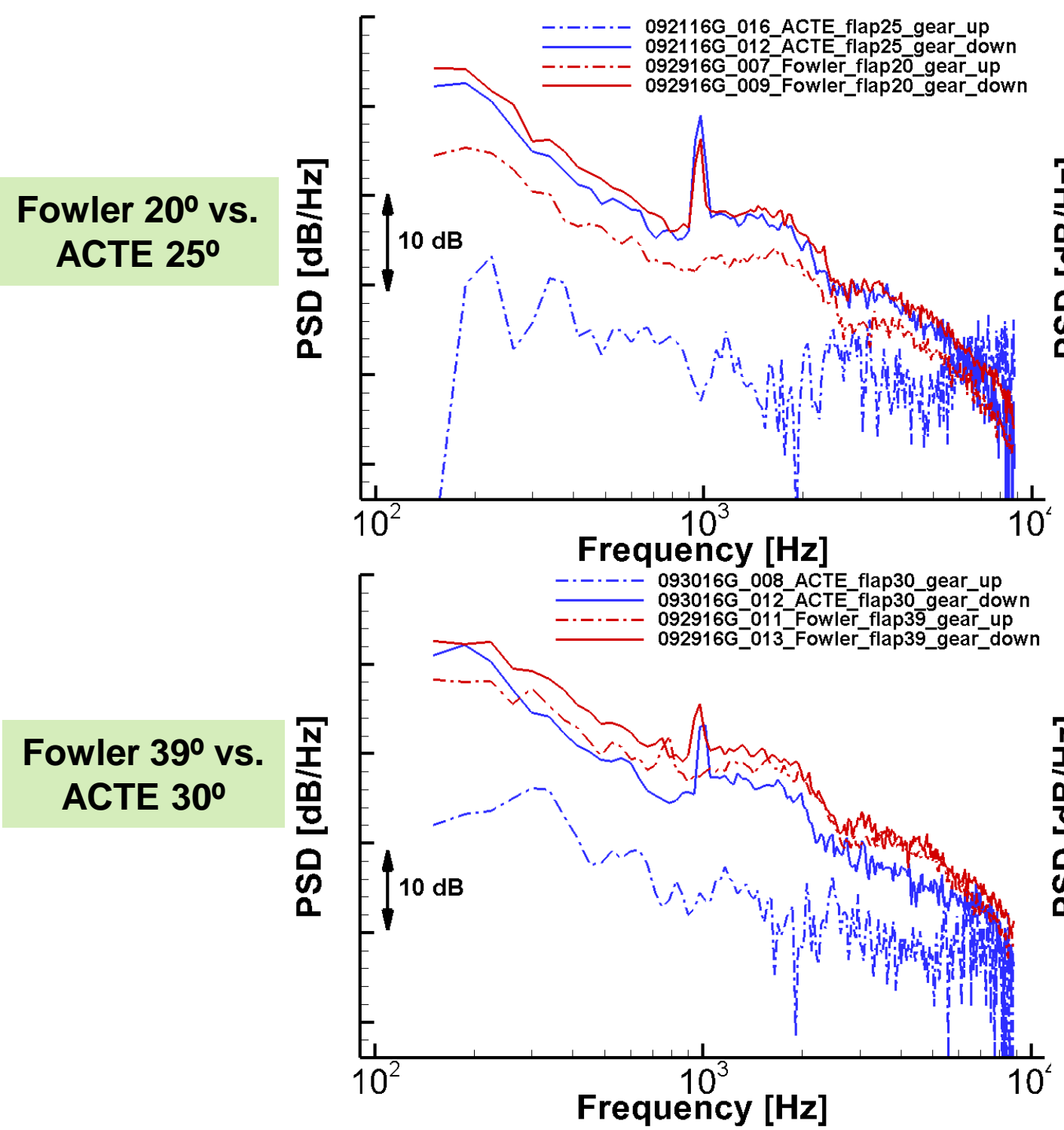

Simulations (array center position)
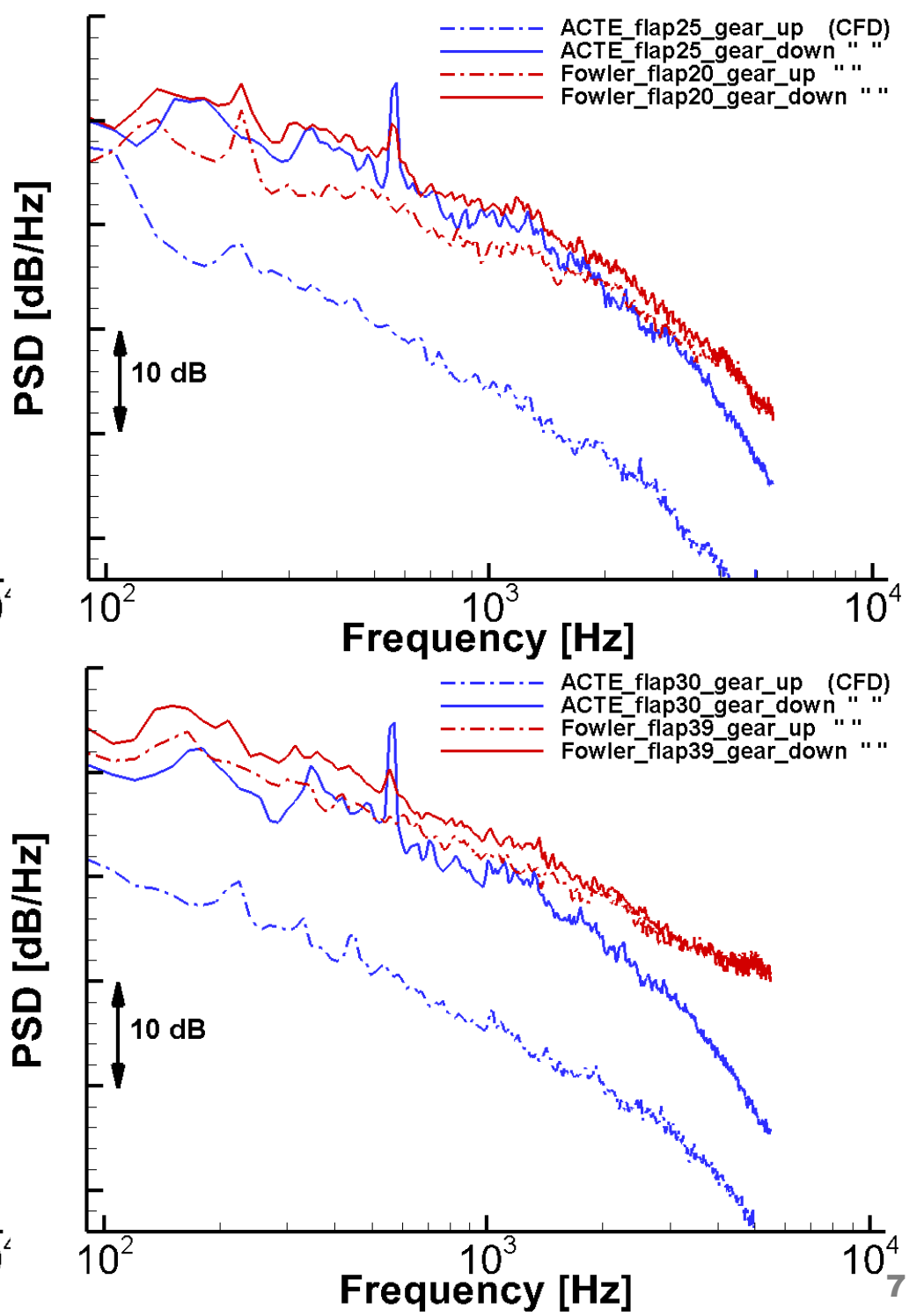


\section{Fine-Resolution Simulation Dataset}

$\square$ Simulation of several key configurations for 804 (ACTE flap) and 808 (Fowler flap) aircraft ongoing

- For 804 aircraft (ACTE flap), conditions for specific passes (mainly AOA) were matched

- Three fine-resolution simulations completed

- Fowler flap 20ㅜ, MLG deployed (808 aircraft)

- ACTE flap 25, MLG deployed without fairings

- ACTE flap 25, MLG deployed with fairings

口 Performance of ACTE flap and MLG fairings compared with noise reduction levels from flight tests

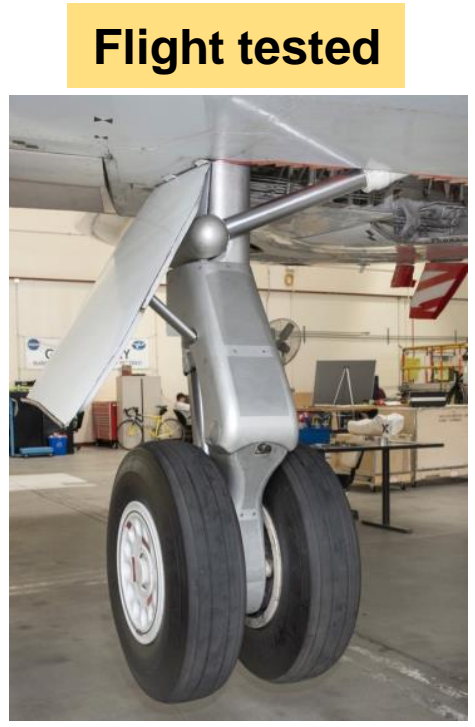

\section{Simulated}

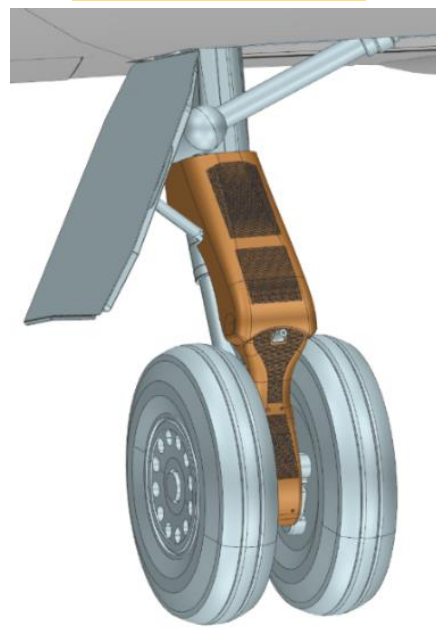

In addition to solid, added a permeable surface with multiple endcaps

- Grid size increased from 7B to 17B voxels

- Substantial increase in computational resources and file sizes

- Volume size enclosed by permeable surface limited frequency resolution to $<2 \mathrm{kHz}$

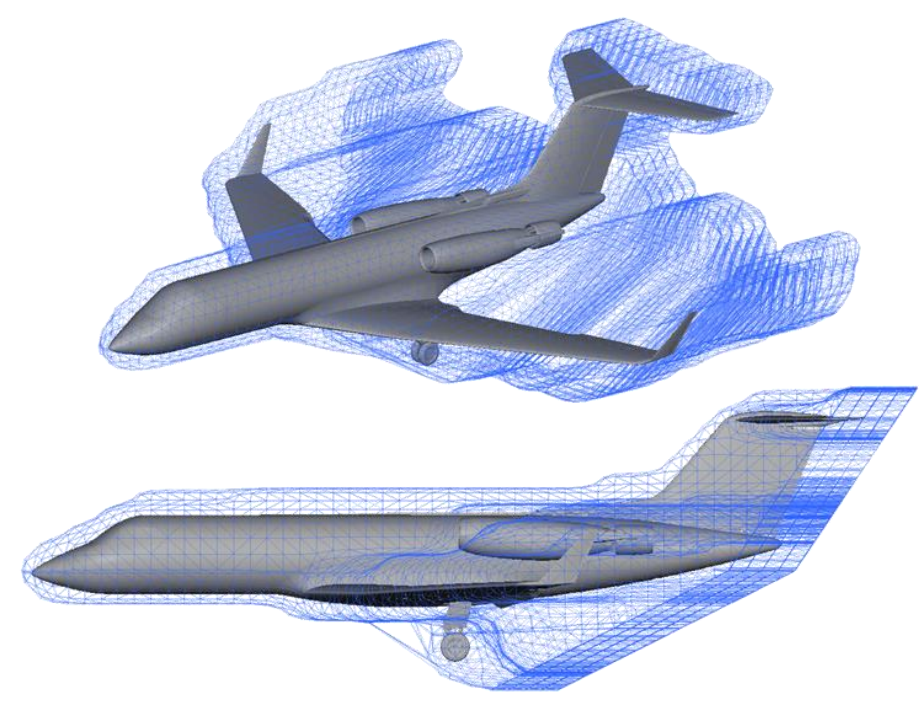




\section{Aerodynamic Comparison}

\section{$\square$ Steady surface pressure}

- ACTE flap 25으, landing gear deployed and retracted
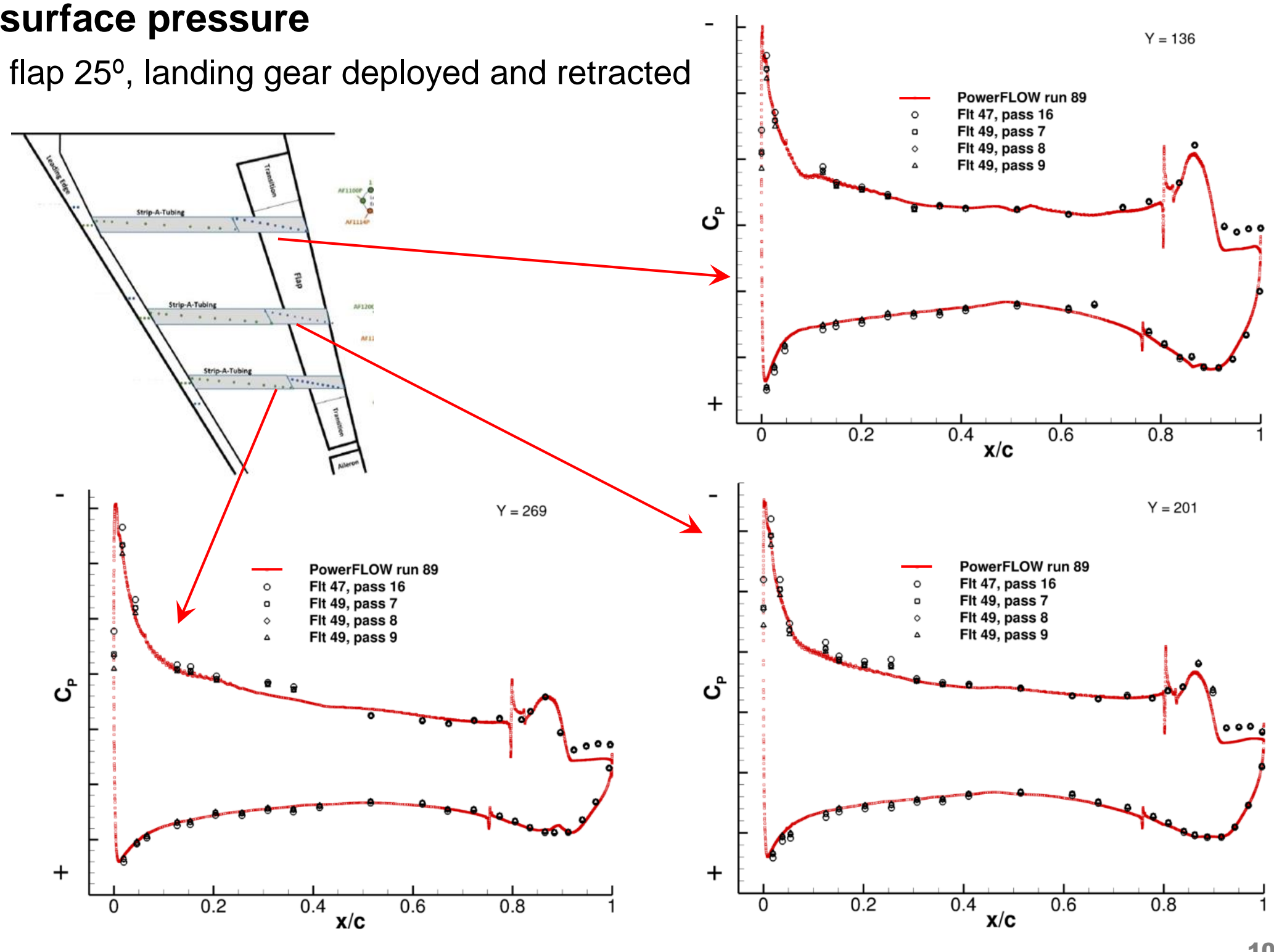


\section{Measured vs. Simulated Beamform Maps}

808 aircraft (Fowler flap 20, landing gear deployed)

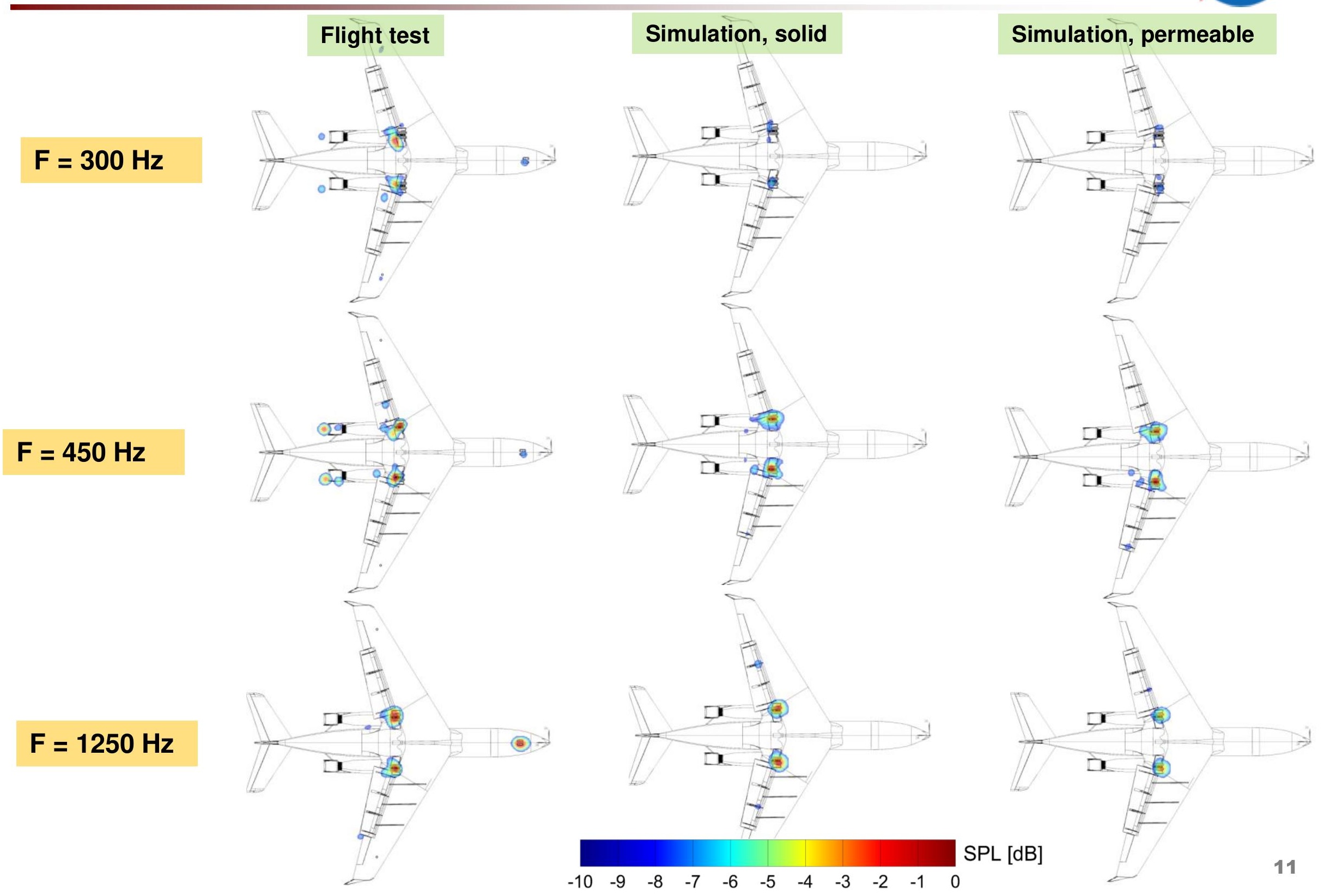




\section{Measured vs. Simulated Beamform Maps}

808 aircraft (Fowler flap 20일 landing gear deployed)

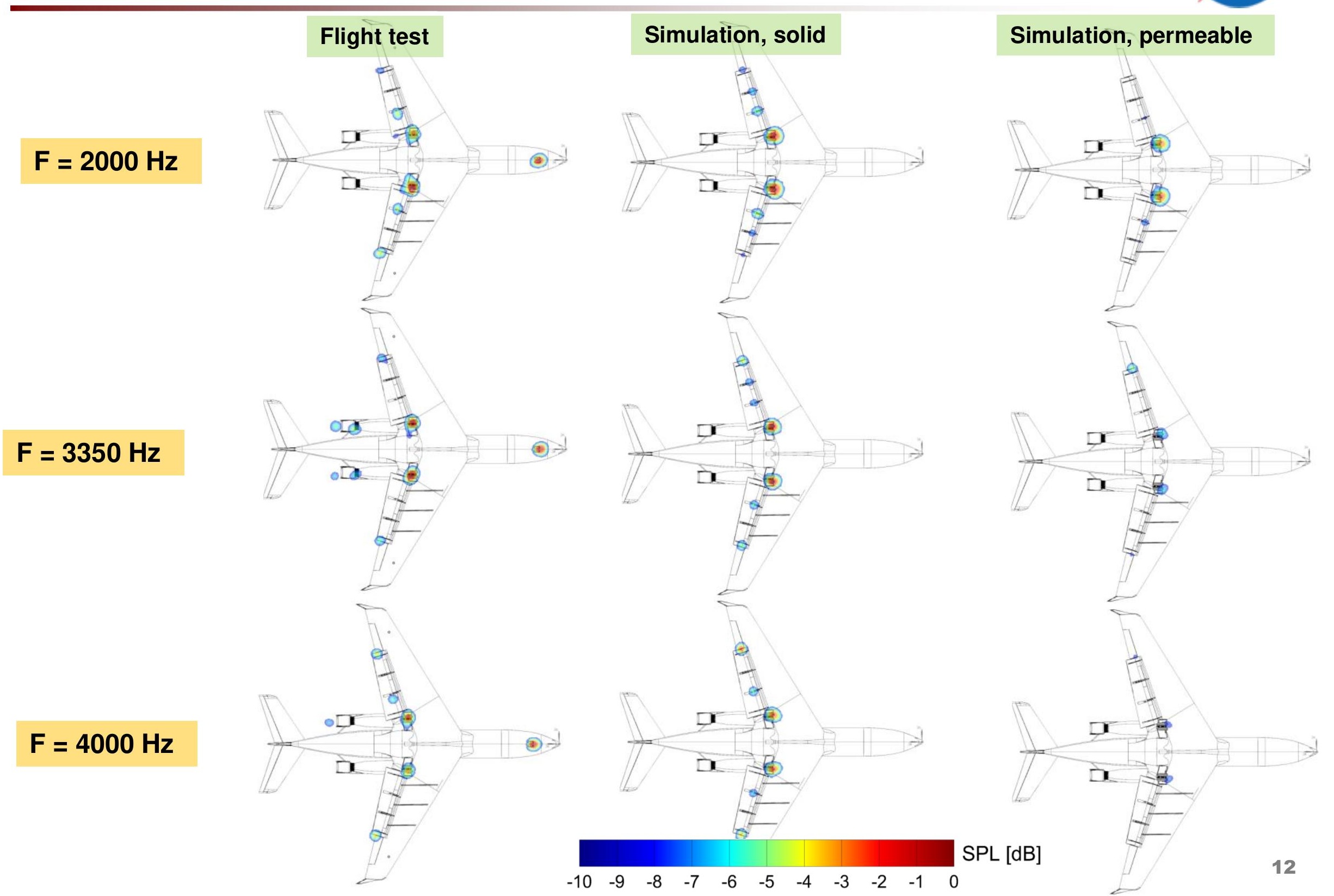




\section{Noise Prediction Trends and Reduction Levels}
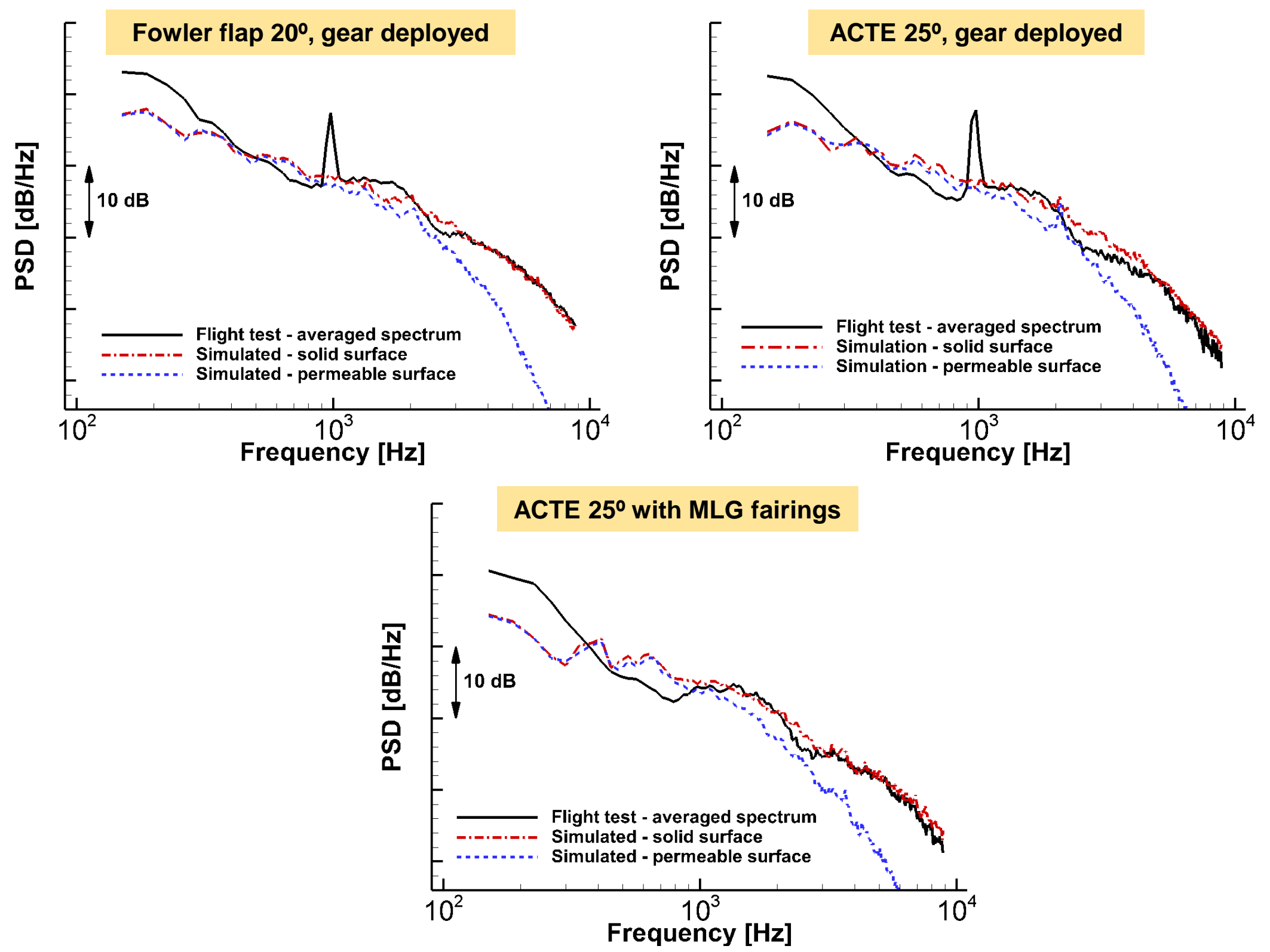


\section{Noise Prediction Trends and Reduction Levels}
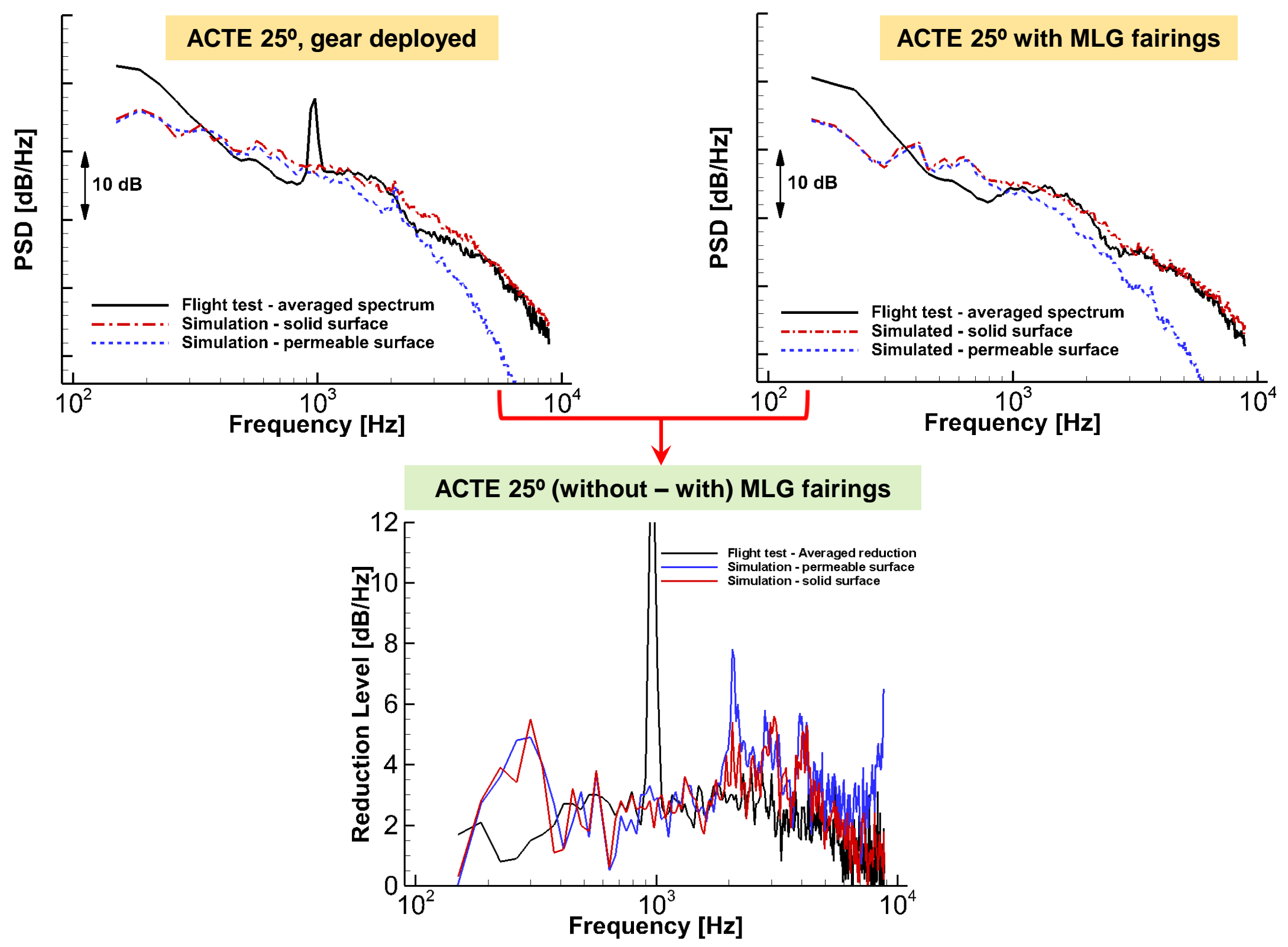


\section{Noise Prediction Trends and Reduction Levels}

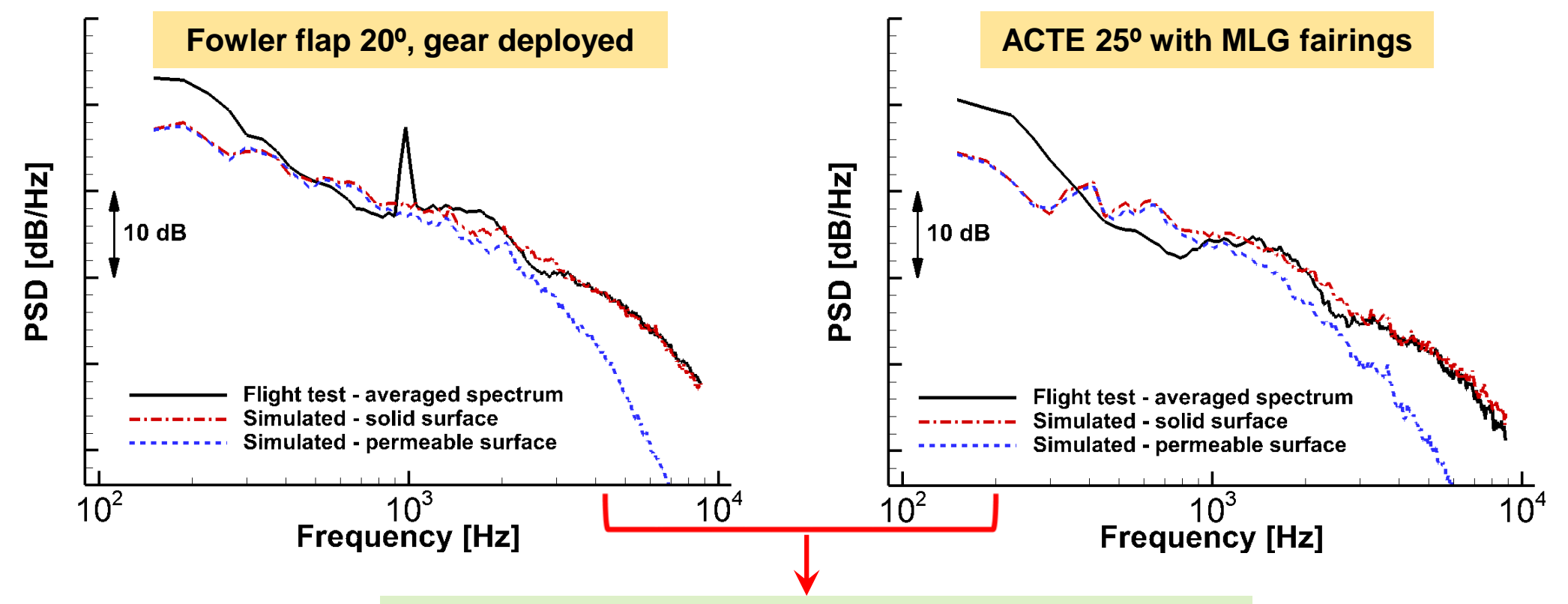

Fowler flap 20 gear down - ACTE 25 with MLG fairings

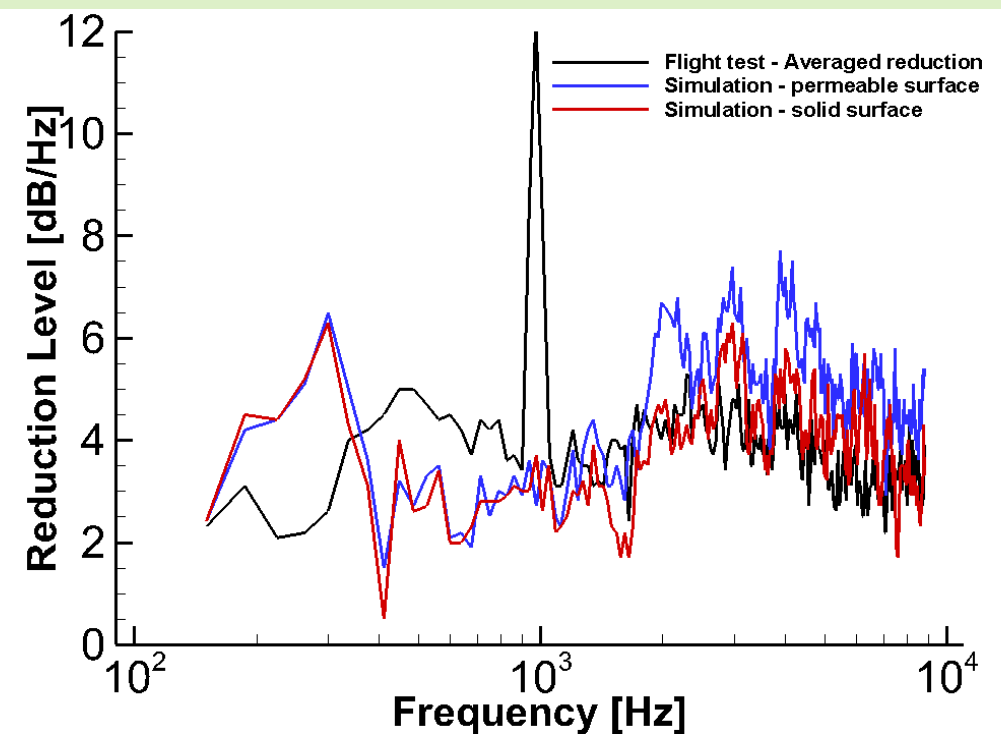




\section{Concluding Remarks}

- Aeroacoustic data from NASA 2016 and 2017 flight tests are being used to assess the predictive capability of companion high-fidelity, full-scale airframe noise simulations

- Blind test simulations with medium spatial resolution properly capture all trends observed in the flight test data

- Predicted steady surface pressures for AOAs matching select flight passes are in excellent agreement with in-flight measurements

- Synthetic array data (solid FWH surface) from fine-resolution simulations with actual inflight conditions are in excellent agreement with measurements for frequencies $>400 \mathrm{~Hz}$

- Integrated farfield spectra (absolute levels)

- Acoustic performance of ACTE flap and MLG fairings (differences in levels)

- As currently modeled,

- Permeable FWH surface results are under-resolved at frequencies $>1.5 \mathrm{kHz}$

- MLG cavity noise, which is dominant at frequencies $<400 \mathrm{~Hz}$, was not captured properly

- Additional, ongoing fine-resolution simulations will permit further validation of computational methodology 


\section{Backup Slides}




\section{Background}

\section{- Issues Facing National Air Transportation System}

- Steady growth in air traffic

- Vital role of air transportation system on US and global economies

- Aircraft noise adversely affects population centers adjacent to major airports

$>$ By far, primary complaint to FAA

- For air transportation to maintain its current expansion path, significant gains in aircraft efficiency and emissions reduction must be achieved

\section{- Aircraft Noise}

- Propulsive (engine)

- Airframe

$>$ Most important during approach

> Broadband and non-compact

> Under-carriage and high-lift devices are prominent noise sources

$>$ Significant reductions in aircraft noise not possible without airframe noise mitigation

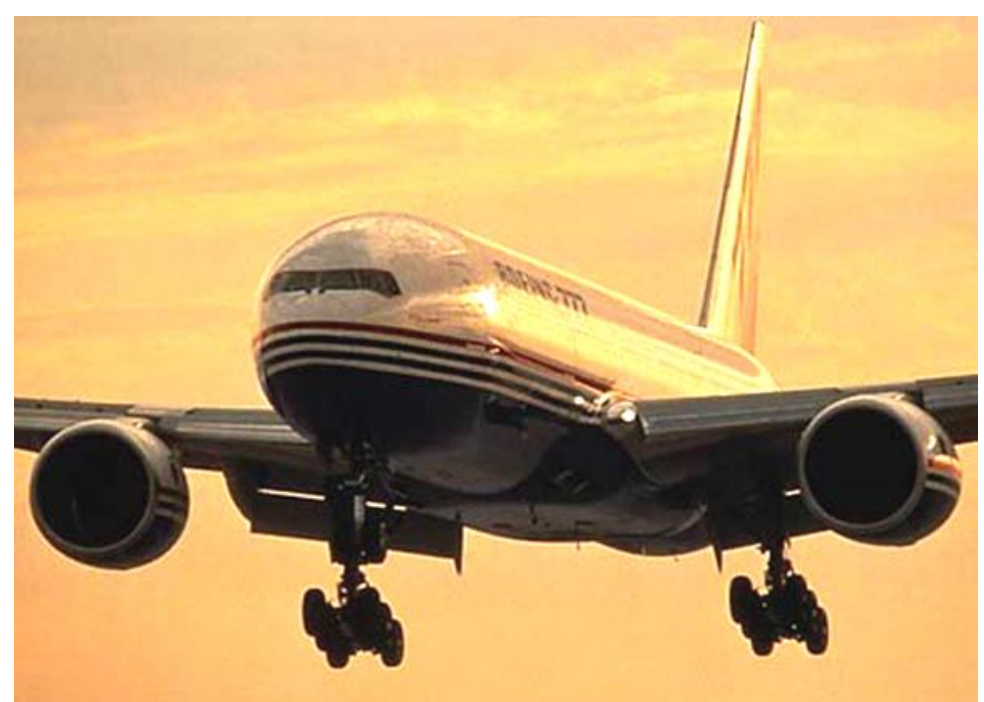

